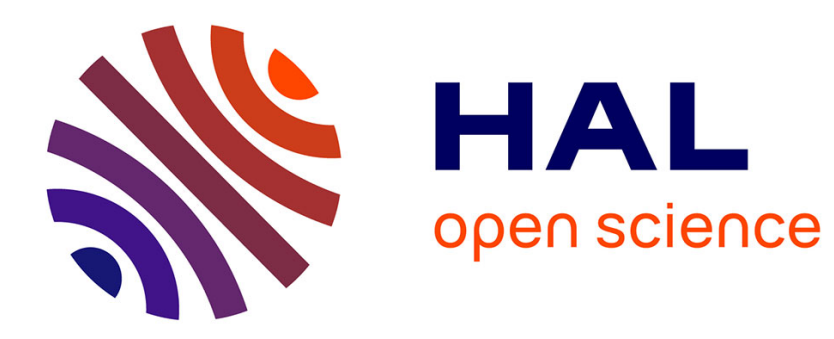

\title{
Les voix du Théâtre du Peuple de Bussang
}

Bénédicte Boisson

\section{To cite this version:}

Bénédicte Boisson. Les voix du Théâtre du Peuple de Bussang. Revue Sciences/Lettres, 2017, 5, 10.4000/rsl.1181 . hal-02488029

\section{HAL Id: hal-02488029 \\ https://hal.univ-rennes2.fr/hal-02488029}

Submitted on 22 Feb 2020

HAL is a multi-disciplinary open access archive for the deposit and dissemination of scientific research documents, whether they are published or not. The documents may come from teaching and research institutions in France or abroad, or from public or private research centers.
L'archive ouverte pluridisciplinaire HAL, est destinée au dépôt et à la diffusion de documents scientifiques de niveau recherche, publiés ou non, émanant des établissements d'enseignement et de recherche français ou étrangers, des laboratoires publics ou privés. 


\section{Les voix du Théâtre du Peuple de Bussang}

Voices in the People's Theatre at Bussang

\section{Bénédicte Boisson}

\section{(2) OpenEdition}

\section{Journals}

Édition électronique

URL : http://journals.openedition.org/rsl/1181

DOI : $10.4000 /$ rsl. 1181

ISSN : 2271-6246

Éditeur

Éditions Rue d'Ulm

Ce document vous est offert par Université Rennes 2

Référence électronique

Bénédicte Boisson, «Les voix du Théâtre du Peuple de Bussang 》, Revue Sciences/Lettres [En ligne], 5 | 2017, mis en ligne le 02 octobre 2017, consulté le 22 février 2020. URL : http:// journals.openedition.org/rsl/1181; DOI : 10.4000/rsl.1181

Ce document a été généré automatiquement le 22 février 2020.

(c) Revue Sciences/Lettres 


\title{
Les voix du Théâtre du Peuple de Bussang
}

Voices in the People's Theatre at Bussang

\author{
Bénédicte Boisson
}

1 Le Théâtre du Peuple - Maurice Pottecher, fondé en 1895 dans les Vosges, à Bussang, petit village de fond de vallée, entendait s'adresser à tout le peuple tout en renouvelant l'art de son temps. Considéré comme l'une des premières réalisations de l'utopie d'un théâtre populaire en même temps qu'une entreprise de décentralisation avant l'heure, il a pour devise " par l'art, pour l'humanité », devise toujours valable aujourd'hui, tout comme les idéaux qui ont guidé sa création.

2 En effet, ce lieu a ceci de caractéristique qu'il a su se transformer dans une continuité remarquable, trouvant à chacune des époques de son développement de nouvelles manières d'actualiser ses principes fondateurs, sans les renier. Ainsi, des traits perdurent des origines à nos jours, qu'il convient de décrire rapidement pour caractériser ce théâtre et rendre compte de sa spécificité et de sa longévité.

Dès les origines, Maurice Pottecher écrit des textes spécifiquement pour ce lieu, le public qu'il vise et ses acteurs. Il considère en effet que reprendre en province les spectacles parisiens n'est pas une solution, et son répertoire, une cinquantaine de pièces composées pour le Théâtre du Peuple, lui permet de distinguer son entreprise tant des formes de théâtre folkloriques ou paraliturgiques jouées alors en plein air, que des spectacles présentés sur le territoire national par les grandes institutions parisiennes. Ce répertoire est joué sans discontinuer jusqu'en 1973, faisant l'objet de reprises très régulières. Il sera ensuite progressivement remplacé par des grands auteurs classiques et modernes, jusqu'à ce que des commandes à l'écriture permettent, au cours de la période la plus récente, de revenir à des textes élaborés spécifiquement pour le Théâtre du Peuple.

Ce théatre propose également un rapport fort à la nature, sans pour autant être un théâtre de plein air, comme se plaît à le souligner son fondateur. En 1895, la première scène est surélevée dans un champ attenant à la propriété familiale, et les spectateurs 
restent debout face à elle. Mais un bâtiment théâtral tout de bois sera progressivement construit. Une voûte, véritable coque de bateau renversée, vient couvrir gradins et tribunes en 1924, mais la scène continue de s'ouvrir sur la nature, grâce à ses portes du fond donnant sur une colline arborée. L'imaginaire wagnérien est fort dans l'élaboration de cette salle, progressivement améliorée au cours du siècle, en collaboration avec les menuisiers-charpentiers du village, et classée depuis 1976 comme monument historique.

Illustration 1 - Le Théâtre du Peuple en 1896.

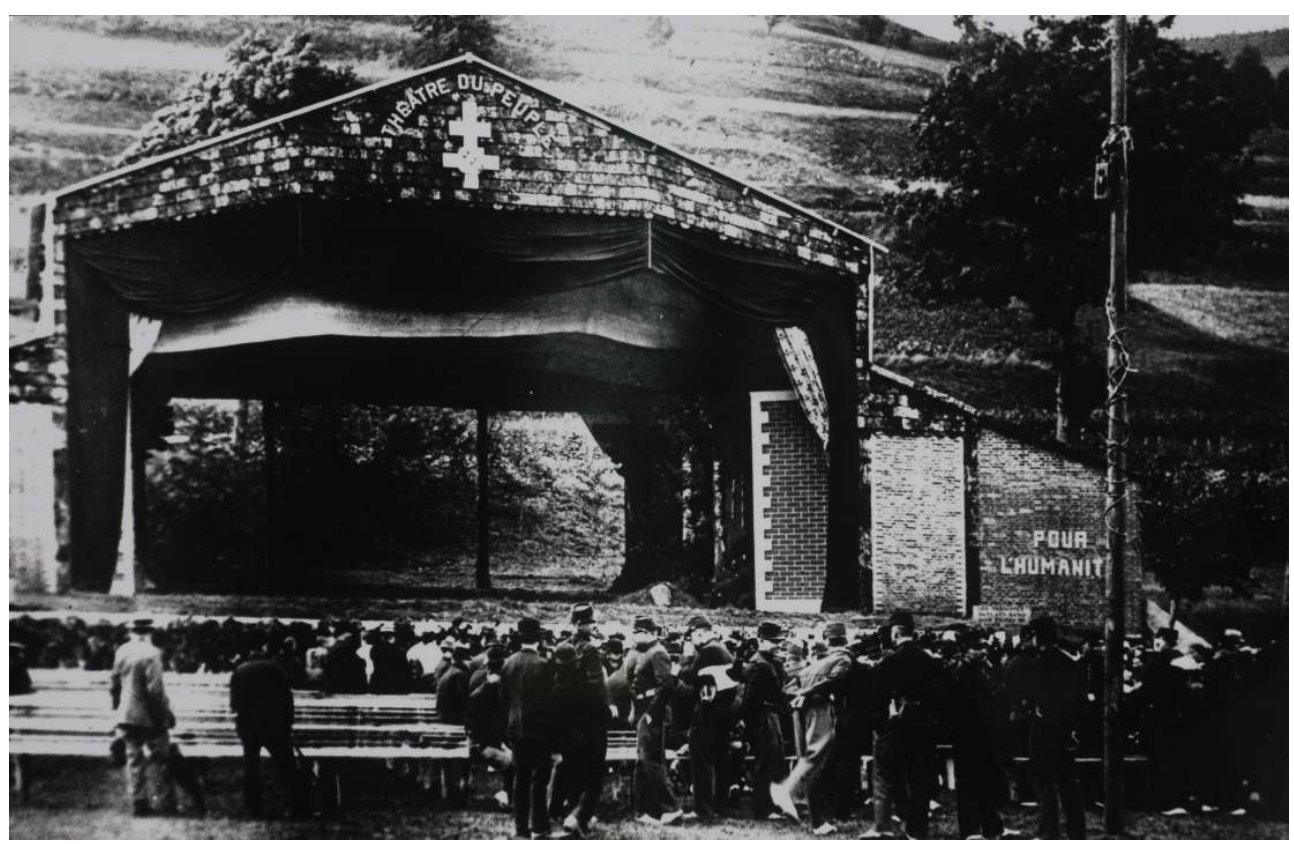

Source : DR/ Archives départementales des Vosges, 83J122/118 - Fonds du Théâtre du Peuple.

5 Une troupe mêlée de comédiens de métier, d'habitants du village et du cercle familial et amical de Maurice Pottecher est présente dès les origines. Ce dernier écrit des rôles pour son épouse, Camille, qui n'est autre que la comédienne Georgette Camée, grande actrice du théâtre symboliste, surnommée par Paul Fort «Camée-à-la-voix-d'or ${ }^{1}$ ». Il fait également jouer des amis artistes et intellectuels, son frère Georges, ses neveux et nièces, les ouvriers de l'usine paternelle et les habitants du village. La troupe doit être désintéressée, refléter la diversité sociale et culturelle attendue dans la salle, et ces acteurs d'un jour présentent pour Maurice Pottecher l'immense avantage de n'avoir été déformés ni par le Conservatoire ni par les tics des acteurs du boulevard parisien ${ }^{2}$.

Enfin, Maurice Pottecher a une conception unanimiste et idéelle du public qu'il entend réunir, conception qui annonce celle de Jean Vilar dans la seconde moitié du $\mathrm{xx}^{\mathrm{e}}$ siècle. Il souhaite s'adresser tant aux personnes parmi lesquelles il a vécu enfant qu'à ses amis artistes et intellectuels parisiens, et décrit ainsi les spectateurs - bientôt qualifiés de « pèlerins de Bussang » - des premières représentations de 1895, venus apprécier Le Diable marchand de goutte :

[L]es blouses se mêlent aux vestons clairs, les chapeaux à fleurs font de l'ombre sur les antiques cornettes : " gens du village », paysans descendus des collines, soldats, officiers, ouvriers, patrons d'usines, familles bourgeoises accourues des bords de la Moselle [...], quelques breaks garnis de touristes et de voyageurs d'été. Ils sont venus au nombre d'environ 2000, ils font un joyeux brou-ha-ha ${ }^{3}$. 
7 La durée de l'engagement dont il a fait et fait encore l'objet pourrait constituer une ultime spécificité de ce théâtre, que ce soit de la part des spectateurs, acteurs amateurs, membres bénévoles de l'association du Théâtre du Peuple - Maurice Pottecher ou même des professionnels. Il n'est pas rare de rencontrer des personnes qui y ont joué pendant plus de trente ans, fréquentent le lieu depuis des décennies ou ont été des membres actifs de la vie du théâtre sur plusieurs générations.

Cette présentation trop rapide ${ }^{4}$ permet de souligner la continuité à l'œuvre au Théâtre du Peuple, tout autant que la singularité de ce dernier. Sa singularité est liée à un développement en marge du territoire mais recherchant une reconnaissance nationale, et à la mise en œuvre d'un projet utopique, visant à concilier ce qui ne cessera de diviser le $\mathrm{xx}^{\mathrm{e}}$ siècle théâtral: amateurs et professionnels, exigence artistique et divertissement populaire, rayonnement national et ancrage local, etc. Si le Théâtre du Peuple est aujourd'hui intégré à la généalogie du théâtre populaire et de la décentralisation théâtrale, il n'a pas pour autant servi de modèle après la Seconde Guerre mondiale, lors de la structuration du réseau de la décentralisation dramatique. Il était alors relativement oublié : ni son projet, liant de manière organique un répertoire, un lieu, une troupe et un public, ni son esthétique, n'ont fait école. Pourtant, une véritable continuité y est à l'œuvre. Lieu de mémoire d'une longévité remarquable, le Théâtre du Peuple a suscité l'investissement de personnes très diverses, qui lui ont permis de perdurer. Fait notable, le couple fondateur du théâtre, ou Pierre Richard-Willm - vedette du cinéma français des années 1930-1940, fils spirituel de Maurice Pottecher, qui a assuré les mises en scène puis la direction artistique du Théâtre du Peuple de 1935 à 1970 -, ont été connus et fréquentés par des personnes qui s'impliquent encore au thêâtre aujourd'hui, et qui évoquent volontiers leurs souvenirs de ces périodes révolues. À Bussang, les temps anciens sont racontés, voire mythifiés et font encore l'objet d'une mémoire directe et fortement valorisée. Par ailleurs, jusqu'au début des années 1970, le répertoire est très stable. On joue les pièces de Maurice Pottecher, en alternant les nouvelles créations et les pièces plus anciennes. Après le décès du Padre, survenu en 1960, le système des reprises devient dominant, favorisant pour les acteurs la transmission des rôles et pour le public, la reconnaissance.

Dans ce lieu qui fut toujours dédié à un théâtre de textes et où - jusqu'à l'abandon du répertoire exclusivement pottechérien - des pièces, un cadre, une troupe et un public sont intrinsèquement liés, il semble que s'organise une mémoire des rôles et de leur interprétation, ainsi que leur passation. La stabilité du répertoire participe de la mise en place de cette mémoire orale, tout autant que la participation à la vie du théâtre dès le plus jeune âge, dans le cadre familial ${ }^{5}$. Ici, comme dans d'autres contextes où coexistent écrit et oral, l'écrit ne supplante pas l'oral et ne supprime pas les problèmes de mémorisation ou le processus d'oralitét. Les textes fournissent des repères, des supports pour se souvenir de rôles qui se constituent aussi par une diction, une intonation, un rythme et une manière propre au Théâtre du Peuple d'interpréter les pièces. Il est révélateur que tous les acteurs interrogés sur leur expérience de ce lieu évoquent d'abord leur voix et la nécessité de se faire entendre ${ }^{7}$. On doit également suivre, au Théâtre du Peuple, le trajet de la voix particulière et célèbre de Georgette Camée - Tante Camm -, rompue au vers libre et au jeu symboliste, qui a formé Pierre Richard-Willm ainsi que la plupart des acteurs ayant foulé les planches du théâtre, jusqu'à son décès en 1957. Ainsi sur la vaste scène de Bussang cohabitaient, grâce à la composition de la troupe et au répertoire choisi, des parlers hétérogènes, plus ou moins formés à une diction théâtrale 
qu'il s'agit de qualifier, reprenant des interprétations et mélodies de jeu déjà entendues, dans un bâtiment à l'acoustique chaude et impure. C'est à dessiner la carte d'identité phonique du Théâtre du Peuple jusqu'aux années 1970, grâce à des sources variées - archives sonores et audiovisuelles, entretiens, films, observation et expérience contemporaine de cette salle -, que nous nous attèlerons ${ }^{8}$.

\section{Les voix célèbres - voix de métier}

"Une parole prononcée sur le diapason ordinaire
n'a pas plus de chance d'être entendue que la voix
d'un poisson dans le fond du Niagara9. "

10 À Bussang, se côtoyaient sur la même scène ouvriers et ouvrières des usines de textile et de couverts du village, paysans, instituteurs, notables, érudits et acteurs de profession, gens du cru ou venus de loin. Parmi eux, la grande Georgette Camée, qui abandonne les expérimentations du Théâtre d'Art pour suivre son mari dans les Vosges ou, ensuite, Pierre Richard-Willm. En 1913, dans le cadre des Archives de la parole sont enregistrées les voix de Camille et Maurice Pottecher, interprétant le prologue de la pièce Liberté $^{10}$, jouée pour la première fois au Théâtre du Peuple en 1898.

Illustration 2 - Liberté, texte et mise en scène de Maurice Pottecher, 1898. Georges Pottecher et Camille Pottecher sur la scène du Théâtre du Peuple, ouverte sur la colline boisée.

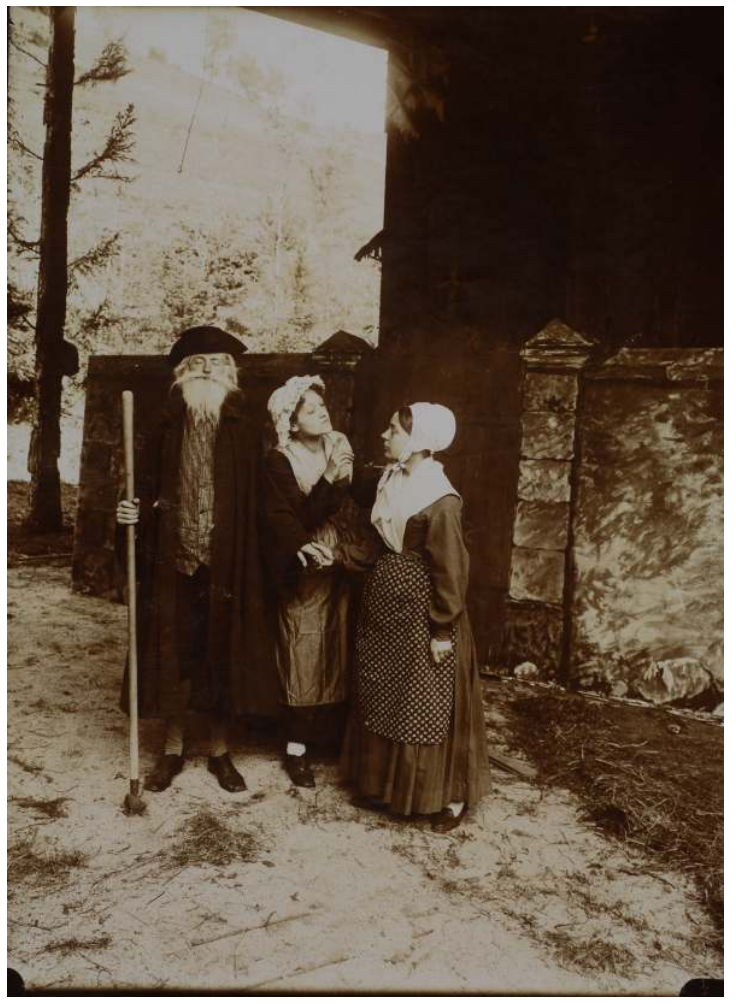

Source : DR / Archives départementales des Vosges, 83J119/10 - Fonds du Théâtre du Peuple.

11 À l'écoute de ce disque, « archi-écriture de l'interprétation ${ }^{11}$ » qui permet d'en étudier les détails tout autant que la matière vocale donnant sa couleur au texte, on perçoit une nette différence entre deux choix de diction : celle d'une actrice, ample, colorée, variée, et 
celle d'un auteur avant tout, plus naturelle, moins timbrée, et dont les effets mélodiques paraissent moins maîtrisés.

Cette différence est à l'image de ce qu'inspirait la voix de Tante Camm. Pierre RichardWillm se souvient dans ses mémoires de sa " voix splendide ${ }^{12}$ ", tandis que les critiques du Théâtre d'Art à Paris furent vite unanimes pour souligner la qualité de sa voix: sa souplesse, sa chaleur, son timbre, mais aussi sa variété. Georgette Camée propose une déclamation nouvelle, inventive, créatrice, loin de la diction classique, qui ravit les poètes symbolistes, ces derniers la considérant comme «la plus subtile trouveuse de lignes et d'intonations qu'il y ait ${ }^{13}$ ». La diction modulée de Georgette Camée se distingue nettement de la voix blanche et du ton monocorde de Lugné-Poe. Comme l'ensemble des comédiens symbolistes, elle participe de ce jeu où l'acteur devient récitant en estompant sa présence physique, pour mieux faire entendre la voix du poème. Mais, à l'instar d'autres comédiennes de ce théâtre, telle Marthe Mellot, Georgette Camée le fait en adoptant un jeu obéissant au "principe décoratif [...], le décoratif s'opposant au mimétique comme le lyrique s'oppose au dramatique ${ }^{14} »$. Il s'agit moins de chercher un effet représentatif que d'élaborer de nouvelles modalités du dire, et de proposer une " mélopée ${ }^{15}$ ", qui travaille sur le mode mélodique plutôt que sur le mode mimétique et mette en avant des cadences et scansions nouvelles, susceptibles de déstabiliser les spectateurs. C'est par ce biais que Georgette Camée quitte l'emphase romantique, pour rendre sensible le rythme de la prose ou du vers libre et élaborer un théâtre de la vocalité. Sur la scène de Bussang, face à cette vaste salle qui ne sera que progressivement couverte, jouant des textes en prose ou en vers libres non dépourvus d'un certain réalisme, on peut imaginer que Georgette Camée ait adapté son jeu et sa puissance vocale. Mais l'enregistrement de Liberté témoigne d'une cadence inhabituelle, d'un jeu mélodique - de plus en plus chanté au fil du passage, en lien avec le contenu même du texte - et d'une amplitude vocale réelle. Il semblerait que la maîtrise de la diction de cette grande actrice ait été transmise à tous ceux qui ont bénéficié de son enseignement. Au Théâtre du Peuple, on parle volontiers de « l'école de Tante $\mathrm{Camm}^{16}$ ", qui a formé à la fois des amateurs importants, telle Marguerite Vannson, ou de grands professionnels comme Pierre Richard-Willm.

13 Après avoir tenu son premier rôle en 1911 à Bussang, joué pendant quinze ans en amateur, puis étayé son expérience avec Ida Rubinstein ou à l'odéon auprès de Firmin Gémier, entre 1925 et 1929, Pierre Richard-Willm commence une carrière au cinéma, où il réussit, aux débuts du parlant, dans les rôles de jeune premier moderne et romantique. Au cours des années 1930, les films se succèdent à vive allure et sa renommée grandissante - déchaînant les passions des "Willmettes »-sera couronnée par la médaille d'or de Pour vous et consacrée avec Le Comte de Monte-Cristo (Robert Vernay, 1942). Au moment où il est approché par les studios de cinéma, Louis Jouvet lui propose également de rejoindre la Comédie des Champs-Élysées. Pierre Richard-Willm, après avoir refusé de travailler avec Baty, Dullin et Pittoëff, décline de nouveau cette offre, jugeant que les spectacles épurés de Jouvet étaient à l'antithèse de ce qu'il faisait et aimait, et s'adressaient à l'esprit plutôt qu'au cœur. Mais les commentaires de Louis Jouvet sur son interprétation d'Antiochus, rapportés par l'acteur dans ses mémoires, permettent de qualifier son type de jeu :

[...] [V]otre diction est sans faille - la vaste scène de Bussang vous a été une bonne école -... et ce n'est pourtant pas commode de «faire le point » entre le trop et le pas assez, à notre époque, dans ces textes d'un autre temps. Côté sensibilité, par contre, vous manquez à mon sens de mesure, de «self control », de discipline ${ }^{17}$. 
14 Si le jeu de Pierre Richard-Willm paraît trop romantique au metteur en scène, qui eût préféré une proposition plus classique, ce témoignage montre bien qu'une maîtrise certaine de la diction s'est transmise de Tante Camm à Pierre Richard-Willm, ainsi qu'une manière de jouer, probablement plus mélodique que naturelle, manquant de mesure au goût de Jouvet. Le témoignage de Nadège Sadon, actrice amateur de Bussang, permet de mieux la caractériser encore. Fille de Marguerite Vannson, elle a commencé à jouer au Théâtre du Peuple à 10 ans, en interprétant en 1948 le rôle de l'un des fils de Mélusine, personnage de Mélusine et son mystère ${ }^{18}$ joué par sa mère. Elle se souvient des répétitions avec Pierre Richard-Willm au cours des années 1950 et 1960, dont elle témoigne ainsi, non sans en sourire :

C'était très curieux, cette façon qu'il avait [de diriger], il lisait le texte, il soufflait en même temps et il indiquait les places. Mais, comment dire, il n'essayait pas de vous faire sortir une émotion ou... il vous disait. Je ne sais pas si c'est une technique du cinéma ou si finalement la Tante Camm faisait la même chose : vous dire une phrase avec les modulations, les intonations. Et vous vous étiez censé... répéter, recopier cela. Ce n'était pas du tout le metteur en scène qui vous parle du texte, qui a étudié le texte, qui vous demande de sortir vos tripes... ${ }^{19}$

\section{Les voix non professionnelles}

Cette évocation des répétitions permet d'aborder le cas des acteurs non professionnels qui avaient acquis une grande expérience de jeu et des voix travaillées, à force de fouler les planches de Bussang, de répéter, d'entendre les pièces jouées et d'être accompagnés par Tante Camm puis par Pierre Richard-Willm. Dans la famille du fondateur, on remarque la truculence de son frère Georges, surnommé le « Raimu lorrain », pour lequel Maurice écrit des rôles qui seront repris par son fils, Frédéric Pottecher, le célèbre chroniqueur judiciaire dont la verve a marqué des générations d'auditeurs de radio. La voix tonitruante d'un magistrat, arrière-neveu de Maurice Pottecher, reste elle aussi dans les mémoires.

On peut supposer qu'à l'instar des autres amateurs, ils ont tous été formés par Camm, qui prenait les comédiens à part pour les faire travailler. À ce sujet, les témoignages se ressemblent: elle ne s'intéressait jamais aux déplacements ni aux gestes. Après avoir réalisé quelques exercices de prononciation et de phonétique, l'apprentissage se faisait «à la becquée » : il fallait répéter après elle, sans se soucier de déployer une émotion. Il s'agissait principalement d'acquérir une voix claire et audible depuis le dernier rang sans qu'aucun effort vocal ne soit perceptible dans la voix. La jauge actuelle de la salle est d'un peu plus de 800 places mais plus de mille spectateurs plutôt agités et bruyants ont pu y être accueillis par le passé :

Je crois que ce qui lui importait, c'est la prononciation, la diction. Elle nous enseignait - je ne sais pas si ça s'enseigne encore dans les conservatoires - la façon de placer sa langue pour prononcer telle ou telle chose. J'ai encore en mémoire, des exercices de ce genre qu'elle me faisait faire. Et puis, bon, vous permettre de parler devant cette immense salle pour qu'on vous entende au fond des tribunes. [...] D'ailleurs, j'ai gardé une voix placée, puissante ${ }^{20}$.

17 Nadège Sadon se souvient ainsi de ses quelques séances avec Tante Camm et se remémore aussi l'avoir vu jouer le rôle de La Mort dans Le Château de Hans ${ }^{21}$ en 1950. Elle en récite une partie au cours de l'entretien, en donnant spontanément le ton, probablement à l'imitation de celui de Camm. Ce rôle, que Camille Pottecher ne souhaitait plus tenir à la 
fin de sa vie, donnera lieu à un enregistrement de sa voix ${ }^{22}$, certainement diffusé lors de la reprise de la pièce en 1957. À la suite du décès de la grande comédienne, Marguerite Vannson interprétera à son tour La Mort, après avoir joué d'autres personnages dans la pièce, notamment le nain jeune, Till ; cette dernière partition sera reprise par sa fille Nadège, quand elle aura pour sa part atteint l'âge d'incarner le nain âgé, Froll.

Illustration 3 - Une représentation du Château de Hans, de Maurice Pottecher, mise en scène, décors et costume de Pierre Richard-Willm, sous la direction de Tibor Egervari et MarieHélène Butel, en 1975.

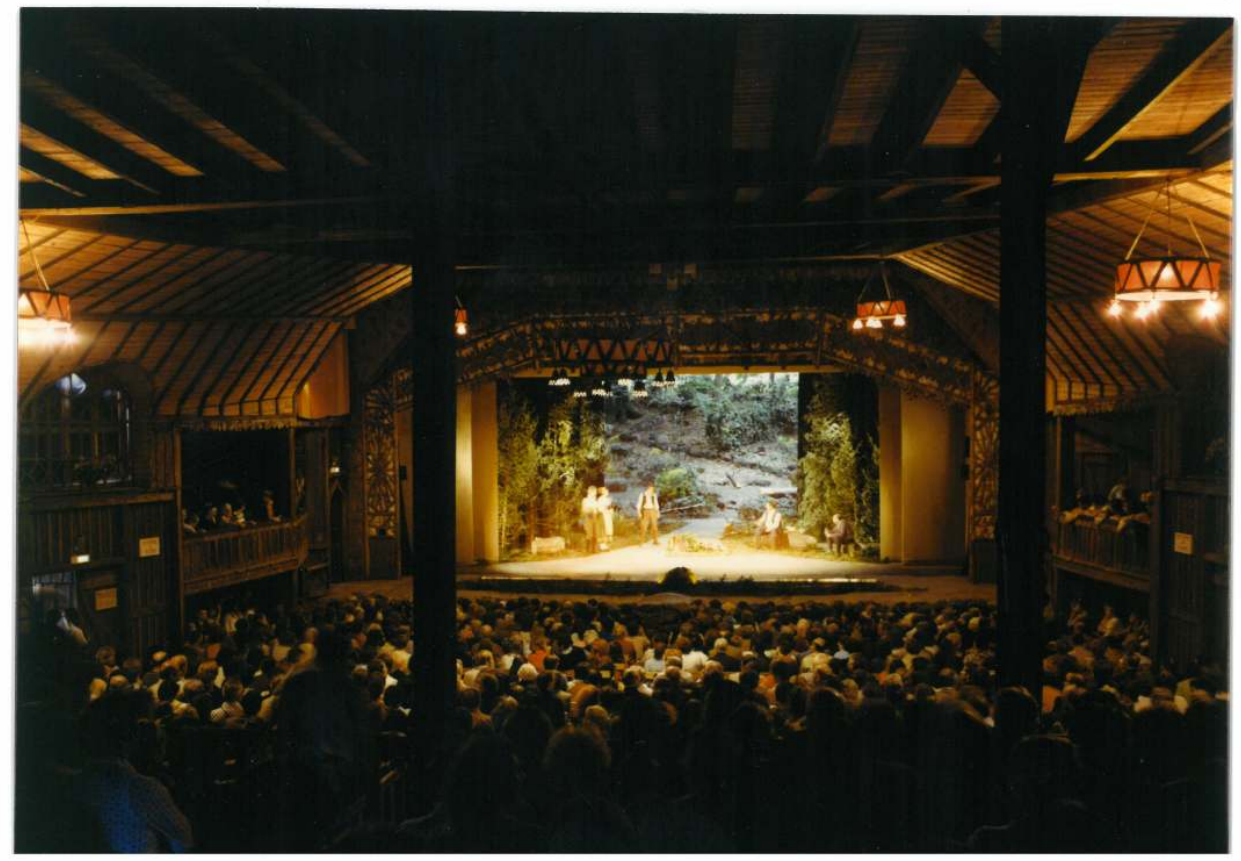

(c) Gabriel Philippe. Fonds du Théâtre du Peuple non déposé.

18 On voit bien comment les rôles se transmettent au gré de la vie des acteurs, mais aussi comment ils sont entendus maintes fois, du plus jeune âge au plus vieux. Tous les comédiens qui ont commencé de jouer à Bussang en amateur, par tradition familiale, racontent des débuts similaires. En été, ils sont au théâtre l'après-midi et aident à tout : ils cousent, peignent, font des accessoires, un peu de figuration et s'amusent. Toute la préparation du spectacle se déroule au théâtre, et dans ce bâtiment de bois tout s'entend. Ainsi chacun, bien qu'occupé à autre chose, peut percevoir les répétitions et apprendre les textes mis en voix. La transmission est donc d'abord orale, les enfants retiennent les pièces en les entendant incarnées par d'autres, en évoluant dans le bâtiment, et en jouant à jouer au théâtre avec les autres enfants de leur âge. La fréquence des reprises permet également une mémorisation certaine. Tout le monde connait tous les rôles par cœur, non seulement le texte, mais certainement aussi le ton: une rythmique, une prosodie, voire une mélodie. On peut ainsi se remplacer au pied levé et se souffler le texte au besoin. Sylvie Tuaillon, actuelle trésorière de l'association et descendante de la famille de charpentiers ayant œuvré à la construction du lieu, raconte avoir passé son enfance au théâtre à prêter main forte pour de multiples tâches, et à craindre qu'un comédien n'oublie son texte. Elle assistait ainsi aux répétitions pour apprendre les pièces par cœur et se les récitait à elle-même, au cours des représentations dont elle était spectatrice ${ }^{23}$. 
19 L'accent et le patois vosgiens, symboles dès les origines d'un théâtre ancré dans un territoire, si ce n'est un terroir, revêtent une importance majeure. Si Tante Camm, en comédienne chevronnée, formée à une diction académique, rectifiait des prononciations et des parlers trop bussenets, Maurice Pottecher pouvait, de son côté, souhaiter que l'accent de la région soit au contraire souligné ou forcé pour certains personnages. Le patois de la Haute Moselle, substitué à celui des paysans de Molière, fut mis à l'honneur dans Le Médecin malgré lui, première pièce présentée à Bussang par Maurice Pottecher en 1892. D'autre part, le répertoire composé par l'auteur se situe souvent dans l'Est, et met en jeu une communauté dans son ensemble, dans sa diversité sociale et culturelle. Cela permet de multiplier les figurants : enfants, jeunes filles et jeunes gens du village ou des montagnes, bûcherons, faneurs et faneuses, paysans, commerçants peuplent le plateau et la scène ouverte. À ceux-là, quand ils ont quelque parole à prononcer, il est souvent demandé de prendre l'accent.

André Bazin, aujourd'hui décédé, ancien ouvrier des usines Pottecher, ouvreur au théâtre de 1947 à 2007 et parfois figurant, évoquait en 2012, avec son parler bussenet, comment on lui avait demandé "de prendre l'accent vosgien » pour le petit rôle de Sulpice, un villageois montant la garde la nuit dans C'est le vent $!^{24}$ en 1954 . Au cours de notre entretien, sa lecture de l'extrait jadis joué, soulignant et grossissant les intonations spécifiques à cette région, donnait une belle idée de son jeu de scène d'alors ${ }^{25}$.

\section{Un répertoire et un cadre spécifiques}

21 Ainsi, les textes de Maurice Pottecher, spécialement créés pour ses acteurs, ses spectateurs et son théâtre, permettent de porter à la scène différentes manières de s'exprimer et jouent des marquages sociaux et culturels des voix, par les thèmes qu'ils abordent et les fables qu'ils proposent. Les amateurs possèdent ces voix diversifiées, qui viennent caractériser leur personnage, sans qu'ils aient à élaborer une interprétation spécifique; il leur suffisait d'accentuer leur prononciation quotidienne. Cela participait de l'effet de réel et d'authenticité recherché par Maurice Pottecher. L'une de ses pièces, Le Sotré de Noël26, créée en 1897, se situe dans les Vosges et son intrigue permet de déployer sur le plateau chants, danses et traditions populaires. Si elle permit de fixer des traditions dont l'auteur avait pu sentir la disparition proche, on peut supposer qu'elle servit également à conserver, ou du moins à faire entendre, des élocutions, des accents et des manières de parler socialement distincts.

Au-delà des personnages, ce sont ainsi des voix et des dictions plurielles qui coexistent sur la scène de Bussang: celles des professionnels et celles des amateurs, celles de diverses provenances régionales, de diverses fonctions, de diverses classes sociales. Cet ensemble hétérogène paraît cependant être traversé par une diction propre au Théâtre du Peuple, héritée de Tante Camm, selon notre hypothèse, mais également liée à l'acoustique du lieu. Cette diction, proche parfois de la déclamation, proposerait une modulation musicalisée de la voix, une modalité décorative, que l'on entend dans un extrait télévisé de L'Anneau de Sakountala ${ }^{27}$, repris à Bussang pour la saison 1960. Au sujet de ce spectacle, qui connaît un réel succès public, un critique de Libération écrivait :

Car L'Anneau de Sakountala, c'est du théâtre comme on n'en fait plus depuis un demi-

siècle...[...] Quant au jeu des « comédiens »... c'est Mounet-Sully au patronage. [...]

Cette critique, non pour médire, mais parce que Bussang pose, une fois de plus, le problème de la culture populaire. [...] 
Il faudrait, là-bas, un Dasté pour installer peu à peu le vrai théâtre. Et refaire, de ce

"Théâtre du Peuple », un théâtre populaire. D'aujourd'hui ${ }^{28}$. la direction de Jean-Louis Barrault en 1942, Julia Gros de Gasquet souligne à quel point le $\mathrm{xx}^{\mathrm{e}}$ siècle théâtral opère une rupture importante. En quelques années, on passe d'une voix travaillée pour être chantante, conçue comme un "organe purement musical ", à une voix qui ne saurait être sa propre fin. Les vers ne sont pas déclamés par Marie Bell, ils sont dits «selon la manière courante de parler ». Ce n'est plus la «beauté de la voix en soi » qui compte, mais la voix comme instrument de la lecture du texte ${ }^{29}$. Si la modalité de jeu entendue à Bussang évoque Mounet-Sully au critique parisien, le jeu de cet acteur ne put servir de modèle à Georgette Camée, qui proposa une diction se détachant à la fois de la diction classique et de l'emphase romantique. Soit le critique, imprécis, fait référence à Mounet-Sully simplement parce que le jeu est déclamé - il aurait alors pu nommer tout autre acteur du XIX ${ }^{e}$ siècle pour insister sur le caractère suranné de cette exécution -, soit Pierre Richard-Willm, alors metteur en scène, a contribué à ajouter une certaine sentimentalité dans l'interprétation. Toujours est-il qu'au Théâtre du Peuple semblait s'être développée une modalité de jeu spécifique, où la voix des acteurs, qu'il s'agisse de celle des amateurs ou de celle des professionnels, était modelée moins par l'incarnation psychologique du personnage que par la nécessité d'être puissante, de proposer une certaine musicalité et de reprendre la mélodie mémorisée des rôles précédemment tenus par d'autres.

Pour conclure, il faut revenir sur le bâtiment exceptionnel dans lequel se jouent ces pièces, bâtiment dont l'architecture générale n'a plus changé depuis 1937, date à laquelle la cage de scène fut rehaussée. À partir de mon expérience contemporaine de cet espace et de la concordance des témoignages des personnes interrogées, je me permets de postuler que les principales caractéristiques actuelles concernant l'acoustique du Théâtre du Peuple étaient présentes depuis que la salle a été couverte par sa voûte de bois. Éric Ruf, qui a joué à Bussang en 1996 alors qu'il était pensionnaire de la ComédieFrançaise depuis quelques années, décrit bien cette dernière. Il évoque admirablement ce que beaucoup d'autres constatent également : ce théâtre n'est pas un violon, les gens y sont bruyants, le bois y grince, on entend la pluie et le vent - que les portes soient d'ailleurs ouvertes ou non. Les comédiens sont souvent amenés à sortir du théâtre dans le grand jour pour passer d'un côté à l'autre de la scène. Ainsi, le Théâtre du Peuple est selon lui un "dehors/dedans ", un "dehors installé », ce n'est pas une boîte préservée mais un lieu " où il y des fissures ", dans lequel le monde entre ${ }^{30}$. Au cours de l'été 2015, pour célébrer les cent-vingt ans du théâtre, des textes de Maurice Pottecher furent de nouveau joués sur la scène du Théâtre du Peuple, pour la première fois depuis 1995. Ils étaient interprétés par des amateurs et mis en scène par les anciens directeurs du lieu. En entendant résonner dans ce cadre, fond de scène ouvert, ce répertoire désormais désuet, Jean-Claude Berrutti, qui dirigea le lieu de 1998 à 2002, disait avoir cerné ce que Maurice Pottecher avait pu rechercher, et qui tenait à un accord entre des voix hétérogènes dans une acoustique impure. 


\section{BIBLIOGRAPHIE}

Albert, Jacques (réal.), Le Théâtre du Peuple de Bussang. Dimanche en France, Paris, ORTF, 7 mai 1961. Bazin, René, entretien avec Bénédicte Boisson et Marion Denizot, Bussang, le 27 août 2012.

Belmont, Nicole et Calame-Griaule, Geneviève (dir.), Les Voies de la mémoire. Cahiers de littérature orale, $\mathrm{n}^{\circ} 43,1998$.

Boileau, Marie-José, entretien avec Bénédicte Boisson et Marion Denizot, Saint-Maurice-surMoselle, le 28 février 2012.

Boisson, Bénédicte et Denizot, Marion, Le Théâtre du Peuple de Bussang. 120 ans d'histoire, Arles, Actes Sud, 2015.

Bourgeois, Alfred et Pottecher, Maurice, Le Sotré de Noël. Farce rustique en 3 actes, Raon-l'Étape, Louis Geisler, 1898.

Dutertre, Jean-François, « La voix parmi les recueils. Ou la voie parmi les écueils », in J.L. Le Quellec, (dir.), Collecter la mémoire de l'autre, [Vouillé], Geste éditions, 1991, p. 10-19.

Gros de Gasquet, Julia, En disant l'alexandrin. L'acteur tragique et son art, $\mathrm{XVII}^{e}-\mathrm{XX}^{e}$ siècle, Paris, Honoré Champion, 2006.

K.D.K, « Plus beau encore que dans Le Grand jeu (1934), Pierre Richard-Willm anime le Théâtre du Peuple de Bussang où l'on joue toujours, hélas !, à la façon de Mounet-Sully », Libération, 3 sept. 1960.

Losco-Léna, Mireille, La Scène symboliste (1890-1896). Pour un théâtre spectral, Grenoble, Ellug, 2010.

Lugné-Poe, Aurélien, « Pour être un acteur aujourd'hui. Conférence de Mr Lugné-Poe », L’Art moderne, $\mathrm{n}^{\circ}$ 12, 24 mars 1895.

Pottecher, Maurice, Théâtre du Peuple de Bussang. $1^{\text {er }}$ spectacle - 1895 : Le Diable marchand de goutte, Paris, Paul Ollendorff éditeur, 1895.

-, Liberté, drame en trois parties. Suivi de Le Lundi de la Pentecôte, comédie en 1 acte, Paris, Louis Geisler, 1898.

-, C'est le vent ! Comédie villageoise en 3 actes, Paris, P. Ollendorff, 1901.

-, Le Château de Hans. Pièce légendaire en 4 actes et 5 tableaux, Paris, Librairie de « Pages libres », 1908.

—, L'Anneau de Sakountala. Légende dramatique en 7 actes d'après Kalidasa, Paris, P. Ollendorff, 1914.

-, Mélusine et son mystère. Pièce légendaire en 3 actes et 10 tableaux, Bussang, Théâtre du Peuple, 1948.

- et Camille Pottecher (voix), Prologue de la liberté, université de Paris, Archives de la Parole, 1913. Disponible sur Gallica : http://gallica.bnf.fr/ark:/12148/bpt6k1310977t.r=maurice\%20pottecher\% 20libert\%C3\%A9?rk=21459;2

Richard-Willm, Pierre, Loin des étoiles. Souvenirs et dessins, Paris, Belfond, 1975.

Ruf, Éric, entretien avec Bénédicte Boisson, Paris, le 8 février 2014. 
Sadon, Nadège, entretien avec Bénédicte Boisson et Marion Denizot, Bussang, le 28 août 2012.

Tuaillon, Sylvie, entretien avec Bénédicte Boisson et Marion Denizot, Bussang, le 3 mars 2014.

\section{NOTES}

1. Paul Fort, cité par M. Losco-Léna, La Scène symboliste (1890-1896). Pour un théâtre spectral, p. 175. Comme le précise l'auteure, la "voix-d'or » de l'époque étant Sarah Bernhardt, Paul Fort, par cette expression, fait de Georgette Camée la Sarah Bernhardt du théâtre symboliste. De son vrai nom Camille de Saint-Maurice, puis Camille Pottecher, Georgette Camée sera vite surnommée Tante Camm au Théâtre du Peuple.

2. Aujourd'hui, la troupe de Bussang continue de mêler chaque été sur scène amateurs et professionnels. Cette spécificité se traduit dans une convention inter-régionale, signée depuis 2000, qui reconnaît le Théâtre du Peuple - Maurice Pottecher comme un lieu de référence national au sujet de la rencontre des pratiques théâtrales en amateur et professionnelles.

3. M. Pottecher, Théâtre du Peuple de Bussang. $1^{\text {er }}$ spectacle - 1895 : Le Diable marchand de goutte, p. I-II.

4. Pour de plus amples informations, voir B. Boisson et M. Denizot, Le Théâtre du Peuple de Bussang. 120 ans d'histoire. Cet article est nourri des recherches menées entre 2012 et 2015 avec Marion Denizot pour préparer cet ouvrage, qu'il s'agisse du dépouillement d'archives déposées et de fonds privés, ou de la cinquantaine d'entretiens que nous avons réalisés avec des membres de la famille du fondateur, des membres de l'association du Théâtre du Peuple - Maurice Pottecher, des acteurs professionnels, des amateurs, des spectateurs, des bénévoles, des membres de l'équipe permanente du lieu et les directeurs qui se sont succédé à sa tête.

5. Les modalités d'apprentissage des textes, dès l'enfance, sont à rapprocher des processus d'apprentissage des littératures orales, décrits pas divers ethnologues et caractérisés de manière synthétique par Geneviève Calame-Griaule et Nicole Belmont: importance de l'écoute, de l'imprégnation, de la répétition et, parfois, rigueur de l'initiation. Voir N. Belmont et G. CalameGriaule, «Les voies de la mémoire. Éditorial » p. 7-24, p. 23-24.

6. Nicole Belmont et Geneviève Calame-Griaule tirent cette conclusion du texte de Frank AlvarezPéreyre sur les versions écrites et orales de la Bible et du Talmud dans la tradition juive, présenté dans le numéro qu'elles dirigent. Voir ibid., ainsi que F. Alvarez-Péreyre, « Le sens de la mémoire et la mémoire du sens ", dans ce numéro, p. 25-45.

7. Est ainsi évoquée une conception de la diction "au sens matériel et physique », qui précède l'expression : la nécessité première est de se faire entendre et de gommer son accent. Cette idée vaut jusqu'au XIX ${ }^{\mathrm{e}}$ siècle note Julia Gros de Gasquet, pour ce qui concerne l'alexandrin. Voir J. Gros de Gasquet, En disant l'alexandrin. L'acteur tragique et son art, XVII $-\mathrm{XX}^{e}$ siècle, p. 20.

8. L'arrêt de cette étude au début des années 1970 s'explique à la fois par la remise en cause de la prééminence du répertoire pottechérien à partir de 1973, qui implique une rupture dans la tradition des reprises, et par l'arrivée d'un nouveau directeur artistique, Tibor Egervari, qui, contrairement à Pierre Richard-Willm, n'a pas été formé à Bussang. Il amène dès lors avec lui une autre tradition, celle de la décentralisation dramatique et du théâtre populaire. Élève de la quatrième promotion du Centre dramatique de l'Est (1957-1960), marqué par les conceptions théâtrales de Michel Saint-Denis, Jean Vilar ou Giorgio Strehler et Paolo Grassi, il revendique l'influence de ses maîtres au CDE, Hubert Gignoux et Pierre Lefèvre, ainsi que le rôle de Pierre Richard-Willm dans son parcours. Tibor Egervari débute à Bussang au cours des années 1960, en assurant la reprise de mises en scène de Pierre Richard-Willm puis assume, de 1972 à 1985, la direction artistique du lieu, tout en étant professeur au département de théâtre de l'université d'Ottawa. 
9. Edgar-Allan Poe, cité par Aurélien Lugné-Poe, dans le cadre d'une conférence intitulée « Pour être un acteur aujourd'hui », dont les éléments essentiels furent publiés dans A. Lugné-Poe, «Pour être un acteur aujourd'hui. Conférence de Mr Lugné-Poe ».

10. M. Pottecher, Liberté, drame en trois parties. Suivi de Le Lundi de la Pentecôte, comédie en 1 acte. Pour l'enregistrement: Maurice Pottecher et Camille Pottecher (voix), Prologue de la liberté, université de Paris, Archives de la Parole, 1913. Disponible sur Gallica: http://gallica.bnf.fr/ ark:/12148/bpt6k1310977t.r=maurice\%20pottecher\%20libert\%C3\%A9?rk=21459;2

11. J.-F. Dutertre, "La voix parmi les recueils. Ou la voie parmi les écueils », p. 19. Ce disque ne rend tout de même pas compte du jeu en scène, dans le cadre du Théâtre du Peuple, avec son public et son acoustique spécifiques.

12. P. Richard-Willm, Loin des étoiles. Souvenirs et dessins, p. 85.

13. Pierre Quillard cité par M. Losco-Léna, op.cit., p.177. Pour le passage précédent, voir les p. 167-177 du même ouvrage.

14. Ibid., op. cit., p. 160.

15. Ibid., p. 168.

16. Marie-José Boileau, entretien avec Bénédicte Boisson et Marion Denizot, Saint-Maurice-surMoselle, 28 février 2012. Marguerite Vannson, ouvrière puis secrétaire de la mairie de Bussang est repérée dès les années 1930 par Tante Camm. Après la Seconde Guerre mondiale, elle tiendra, des années 1940 aux années 1960, de très grands rôles au Théâtre du Peuple. Ses filles, MarieJosé Boileau et Nadège Sadon s'investissent elles aussi au théâtre, où elles aident et débutent comme actrices dès leur plus jeune âge. La première est aujourd'hui encore membre de l'association du Théâtre du Peuple-Maurice Pottecher tandis que la seconde a assuré l'administration du théâtre à la suite de sa mère (1973-1991) tout en partageant parfois la scène avec cette dernière et en reprenant progressivement un certain nombre de ses rôles.

17. P. Richard-Willm, op. cit., p. 177.

18. M. Pottecher, Mélusine et son mystère. Pièce légendaire en 3 actes et 10 tableaux.

19. Nadège Sadon, entretien avec Bénédicte Boisson et Marion Denizot, Bussang, le 28 août 2012.

20. Nadège Sadon, id.

21. M. Pottecher, Le Château de Hans. Pièce légendaire en 4 actes et 5 tableaux.

22. La bande de cet enregistrement a été retrouvée dans les archives du Théâtre du Peuple et est dorénavant déposée aux Archives départementales des Vosges. Nous n'avons pas encore pu l'écouter puisque les appareils permettant de le faire n'existent plus. Il faut désormais attendre qu'une version numérique de ce document soit produite.

23. Sylvie Tuaillon, entretien avec Bénédicte Boisson et Marion Denizot, Bussang, le 3 mars 2014.

24. M. Pottecher, C'est le vent ! Comédie villageoise en 3 actes.

25. René Bazin, entretien avec Bénédicte Boisson et Marion Denizot, Bussang, le 27 août 2012.

26. A. Bourgeois et M. Pottecher, Le Sotré de Noël. Farce rustique en 3 actes.

27. M. Pottecher, L'Anneau de Sakountala. Légende dramatique en 7 actes d'après Kalidasa. La pièce est jouée pour la première fois au Théâtre du Peuple en 1922. Pour l'extrait filmé de la pièce, voir J. Albert (réal.), Le Théâtre du Peuple de Bussang. Dimanche en France.

28. K.D.K, « Plus beau encore que dans Le Grand jeu (1934), Pierre Richard-Willm anime le Théâtre du Peuple de Bussang où l'on joue toujours, hélas !, à la façon de Mounet-Sully. »

29. Pour tout ce passage, voir J. Gros de Gasquet, op. cit., respectivement p. 244 et p. 246.

30. Éric Ruf, entretien avec Bénédicte Boisson, Paris, le 8 février 2014. 


\section{RÉSUMÉS}

Cet article tente de dresser le portrait phonique du Théâtre du Peuple - Maurice Pottecher, situé à Bussang, dans les Vosges, entre sa fondation en 1895 et 1973. Connu dans l'histoire du théâtre comme l'une des premières tentatives de théâtre populaire et décentralisé, ce projet utopique tenta, dès ses origines, de s'adresser à tous, en articulant un répertoire spécifique, une troupe mêlant amateurs et professionnels, et un bâtiment de bois ayant la particularité de s'ouvrir sur la nature. Dans ce théâtre, la mémoire des rôles s'est transmise de manière orale. L'épouse de Maurice Pottecher, qui fit des débuts remarqués dans le théâtre symboliste parisien, eut une influence forte dans le mode de diction qui fut adopté au Théâtre du Peuple, alors même que des parlers hétérogènes y étaient entendus, reflétant la diversité sociale, culturelle et de formation de ceux qui y jouaient. Cet article s'intéresse donc à l'histoire de la diction dans le théâtre français, aux voix des amateurs et des professionnels, aussi bien qu'à l'acoustique des salles.

This article tries to draw the vocal portrait of the People's Theatre - Maurice Pottecher, located at Bussang, in the Vosges, from its foundation in 1895 to 1973. Famous in the history of theatre as one of the first experiments in popular, decentralized theatre, this utopian project from the very beginning tried to appeal to everyone and joined a specific repertoire, which mixed amateur and professional comedians, to the use of a wooden building having the distinctive feature of opening onto nature. In this theatre, the memory of roles has been transmitted orally. Maurice Pottecher's wife, who made noticed débuts on the Symbolist stage in Paris, exerted a strong influence on the mode of diction adopted in the People's Theatre, where heterogeneous speeches were heard which mirrored the diversity of the players, socially, culturally and in training. This article thus focuses on the history of diction in French theatre, on the voices of the amateurs and professionals, as well as on the theatres' acoustics.

\section{INDEX}

Mots-clés : diction, histoire du théâtre, amateurs, Théâtre du Peuple, mémoire orale

Keywords : diction, history of theatre, amateurs, People's Theatre, vocal memory

\section{AUTEUR}

\section{BÉNÉDICTE BOISSON}

Maître de conférences en études théâtrales, directrice du département Arts du spectacle, Université Rennes 2 - Université Bretagne-Loire, EA 3208 « Arts : pratiques et poétiques ». Parmi ses publications :

« Dans la lignée d'Artaud : les modalités sonores de la coprésence dans Le Livre de Christophe Colomb (Claudel, Barrault, 1953), Oh les beaux jours (Beckett, Blin, 1964) et Paradise Now (Living Theatre, 1968) », in J. Bovet, J.-M. Larrue et M.-M. Mervant-Roux (dir.), Théâtre/Public. Voix Words Words Words, n 201, juil.-sept 2011, p. 77-82. 
L'Annuaire théâtral. Héritages et filiations du théâtre populaire, avec Marion Denizot (dir.), ${ }^{\circ} 49$, Ottawa, université d'Ottawa, CRCCF, juin 2012, p. 9-152.

«Les compositions sonores au théâtre » avec Éric Vautrin, in C. Guiu et al., (dir.), Soundspaces. Espaces, expériences et politiques du sonore, Rennes, PUR, 2014, p. 31-40.

Le Théâtre du Peuple de Bussang. Cent vingt ans d'histoire, avec Marion Denizot, Arles, Actes Sud, 2015.

La Mise en scène théâtrale de 1800 à nos jours, avec Alice Folco et Ariane Martinez, Paris, PUF (Licence), 2010, réédition PUF (Quadrige), 2015. 